\title{
JUVENILE NASOPHARYNGEAL ANGIOFIBROMA: OUR EXPERIENCE
}

Prasanna Kumar S1 ${ }^{1}$ A Ravikumar ${ }^{2}$, Somu L ${ }^{3}$, Srinivasa Reddy ${ }^{4}$

\section{HOW TO CITE THIS ARTICLE:}

Prasanna Kumar S, A Ravikumar, Somu L, Srinivasa Reddy."Juvenile Nasopharyngeal Angiofibroma: Our Experience". Journal of Evolution of Medical and Dental Sciences 2014; Vol. 3, Issue 03, January 20; Page: 559564, DOI:10.14260/jemds/2014/1865

ABSTRACT:BACKGROUND:Juvenile nasopharyngeal angiofibroma (JNA) is an uncommon lesion which exclusively occurs in an adolescent male. They comprise about $0.05 \%$ to $0.5 \%$ of all head and neck tumors. The surgical management of this lesion is challenging. This tumor being highly vascular has the potential to bleed torrentially during surgery. The advent of endoscopic sinus surgery has significantly modified the way in which the tumors of the nose and paranasal sinus have been managed.AIM AND OBJECTIVES OF THE STUDY:Audit the clinical and operative data, to ascertain the role of preoperative embolization and autologous blood transfusion.STUDY DESIGN: Retrospective study.SUBJECTS AND METHODS:Clinical and operative data of all patients of JNA during the period 2008 to 2011were included in this study.RESULTS: Complete tumor removal was obtained in all the eight cases. Seven of these cases had Stage II b and 1 had stage III b (Radkowski classification). Six cases were managed by endonasal endoscopic approach. Two cases were treated by external approach. All patients underwent super selective embolisation preoperatively 24 hour prior to surgery. The average blood loss during surgery was $362 \mathrm{ml}+/-176.2 \mathrm{ml}$. All but one had received autologous blood transfusion during surgery. CONCLUSION: Pre-operativeembolization minimizes the intra operative blood loss. Autologous blood transfusion needs to be planned in advance and reduces the morbidity associated with transfusion. Endonasal endoscopic surgery has tremendously reduced the morbidity and facial morphological deformity in surgical management of Juvenile nasopharyngeal angiofibroma.

KEY WORDS: Juvenile nasopharyngeal angiofibroma, intracranial extension, embolisation, endoscopic approach.

INTRODUCTION: It was Chelius (1847) 1 who first recognized that Juvenile nasopharyngeal angiofibroma as a fibrous polyp that "commonly occurs in persons at the time of puberty".Legouest (1865) ${ }^{1-4}$ reported the correlation of these fibrous polyps and their occurrence in adolescent males. Goosselin (1876) ${ }^{1}$ noted the tendency of these tumors to regress after puberty. Chaveau (1906) $1,3,4$ used the term juvenile nasopharyngeal angiofibroma (JNA) to describe this highly vascular fibrous polyp that occurred in adolescent male.

JNA are rare, benign, highly vascular and locally aggressive tumors seen exclusively in adolescent males. They comprise $0.05 \%$ to $0.5 \%$ of all head and neck neoplasm's. They are seen in young adolescent males 1,3 .

There are several staging systems that have been proposed for JNA, these include Sessions (1981) ${ }^{5}$, Fisch (1983) 6, Chandler (1984) 7, Andrew (1989) ${ }^{8}$, andRodkowski (1996) ${ }^{9}$.We have used the Rodkowski staging system in our study.

The excision of juvenile nasopharyngeal angiofibroma is a surgical challenge. Intra operative blood loss has always been a main concern in the past. With the advent of pre- 
operativeembolisation, the excessive intra operative blood loss and the need for post operative transfusion have been reduced.

Various techniques have been described for the complete excision of JNA. Endonasal endoscopic approach has changed the way in which angiofibromas are being managed. We review our experience in dealing with JNA with the aim of auditing the clinical and operative data, to ascertain the role of preoperative embolization and autologous blood transfusion.

METHODS: This retrospective study was done at a tertiary care centre during the period 2007 to 2011.Clinical and operative data of all patients who were diagnosed and managed by us with histopathological confirmation of JNA were analyzed for normative data, presenting symptoms, investigation, staging (Rodkowski), pre-operativeembolization, operative time, blood loss, blood transfusion, and operative time, intra operative and post operative complication.

RESULTS: Eight cases of JNA and were managed successfully during this period of study. All of them were adolescent males ranging from 12yrs to 19yrs of age (average 14.5+/-2.26). Five cases presented to us with recurrent episodes of epistaxis, two came with nasal block and one case presented with proptosis of left eye.

Nasal endoscopy showed unilateral smooth surfaced mass in the nasal cavity in all cases (Fig 1 \& 2). Biopsy was not attempted as our clinical suspicion and diagnosis was JNA. Contrast enhanced CT scan of the paranasal sinuses was done for all cases. We had done MRI for one case in which there was intra cranial extension. All tumors showed marked contrast enhancement and were staged according to Rodkowski classification. The tumor was limited to the pterygomaxillary fissure (stage II B) in six cases (Fig 3 \& 4). One of the case had extension into maxillary sinus, ethmoids, infratemporal fossa, sphenoid sinus and intracranial extension (anterior and middle cranial fossa) (Fig 5, 6 \& 7) without involvement of cavernous sinus (stage III B). Six patients underwent endoscopic removal of JNA. In two cases, the tumor was excised by the transpalatine approach (Wilson approach) and lateral rhinotomy (Fig 8a \& b) (Shaheen's approach) respectively. Pre operative Digital subtraction angiography with super selective embolisation was done for all cases $24 \mathrm{hrs}$ prior to surgery. The average surgical time taken was $187.5+/-50$ minutes. Endoscopic excision took lesser time than external approach. The average blood loss during surgery was 362.5 +/- $176.7 \mathrm{ml}$. Seven cases received 1 pint of autologous blood transfusion during surgery.

DISCUSSION: Juvenile nasopharyngeal angiofibroma are uncommon, highly vascular tumors comprising less than $0.05 \%$ to $0.5 \%$ of all head and neck tumors 1,3 , and 4 . Tumor mostly occurs in adolescent males ${ }^{1-4}$. All cases in our study were also adolescent males. The most common clinical presentation is unilateral, profuse, unprovoked epistaxis. Other symptoms were headache, nasal block, proptosis, facial deformity, diplopia depending on the extent of the tumor.

A high degree of suspicion based on the clinical presentation along with the diagnostic imaging in the form of CT scan and MRI will aid in the diagnosis, staging and management of JNA ${ }^{10}$.

Diagnostic imaging is performed prior to any invasive procedure and it reduces the risk of excessive bleed associated with biopsy. The characteristic findings on radio imaging, is a soft tissue density lesion in the nasopharynx or nasal cavity, a soft tissue lesion in the pterygopalatine fossa, erosion of posterior osseous margin of the sphenopalatine foramen extending to the base of the 
medial pterygoid plate, widening of the sphenopalatine foramen, bowing of the posterior wall of the maxilla. Since this tumour is highly vascular it shows marked contrast enhancement.CT scan is used to identify bony involvement and tumour spread.MRI is useful to show soft tissue differentiation between tumour and surrounding tissue, it also helps in delineating dural involvement in tumour with intracranial extension. MRI also helps discern between sinus invasion, obstruction and retention of secretions. On MR imaging, JNA appears as a heterogeneous mass with signal voids that are consistent with the highly vascular tumour ${ }^{11 .}$

This tumour primarily derives its blood supply from the distal branches of the internal maxillary artery i.e.the sphenopalatine, descending palatine and posterior superior alveolar artery and its branches. As the tumour grows it receives blood supply from the internal carotid system and also from the vessels on the opposite side ${ }^{12}$.

Pre operative angiography was done for all our patients $24 \mathrm{hr}$ prior to the planned surgery. The feeding vessels to the tumor were identified and super selective embolisation was done in the same sitting. We have found that super selective embolisation 24 hour prior to surgery reduces intraoperative blood loss and also provides a better field during dissection.There are very few studies on the ideal timing of embolisation. Though many authors suggest embolisation to be done $24 \mathrm{hrs}$ to $72 \mathrm{hr}$ prior to surgery ${ }^{12}$, no definite prospective study is available in this regards. It is believed that embolisation when done $72 \mathrm{hr}$ or more prior to surgery is ineffective and results in excessive bleeding, because the tumor is revascularised by other collateral vessels.

The treatment goal for JNA is complete surgical excision with minimal morbidity, which is a surgical challenge. Various surgical approaches have been described based on the tumor extent. The approaches include endonasal endoscopic, transpalatine, medial maxillotomy (via degloving or lateral rhinotomy), and Le Fort I, and infratemporal approaches. Tumors confined to the nasopharynx, nasal cavity, and paranasal sinuses may be removed exclusively by endonasal endoscopic approach. Lateral rhinotomy is recommended for tumors that extend to the intratemporal fossa, intracranial cavity or cavernous sinus. In one case we used the Shaheens 13 approach via a Weber Fergusson incision for the excision of tumor.

Autologous blood was transfused for all our patients except one. Blood was collected from the patients 72 hours prior to the surgery and the patient was supplemented with oral hematinic. During surgery the collected blood was transfused back to the patient. Packed cells were also transfused depending on the blood loss. The risk of transfusion related reactions, transfusion transmissible disease and complications are less for autologous transfusion. Teddy Charles Adias et al 14 have recommended the use of 'autologous transfusion should form part of a strategy to minimize the risk associated with allogeneic transfusion.

External radiation therapy (either conventional radiotherapy or Gamma-knife) is usually reserved for patients who present with unresectable intracranial disease or whose clinical condition prevents surgery. Chemotherapy is reserved for recurrences or in cases in which neither surgery nor radiotherapy is possible.

CONCLUSION:All suspected JNA should have imaging studies done prior to any form of surgical intervention. Pre-operative embolization done 24 hours prior to surgery reduces the vascularity of the tumor thereby minimizing the intraoperative blood loss. Autologous transfusion needs to be planned in advance and should be a part of the strategy in managing cases of JNA, thus reducing the 
morbidity associated with allogeneic transfusion. Endonasal endoscopic surgery has tremendously reduced the morbidity and facial morphological deformity in managing cases of Juvenile nasopharyngeal angiofibroma and should be judiciously used whenever it is possible.

\section{REFERENCES:}

1. Maurice Schiff MD, Juvenile nasopharyngeal angiofibroma a theory of pathogenesis; The Laryngoscope, August 1959; Vol LXIX: 981-1016.

2. Schiff, M. (1959), Juvenile Nasopharyngeal Angiofibroma: A theory of pathogenesis. The Laryngoscope, 69: 981-1016. doi: 10.1288/00005537-195908000-00001

3. S. L. Sellars, Juvenile Nasopharyngeal Angiofibroma. S. Air. med. J. 58, 961 (1980).

4. Hayes Martin, Harry E. Ehrlich, Jules C. Abels, Juvenile Nasopharyngeal Angiofibroma,Ann Surg. 1948 March; 127(3): 513-536

5. Sessions RB, Bryan RN, Naclerio RM, Alford BR. Radiographic staging of juvenile angiofibroma. Head Neck Surgery 3:279-283, 1981.

6. Fisch U. The infratemporal fossa approach for nasopharyngeal tumors. Laryngoscope. 1983; 93:36-44.

7. Chandler JR, Goulding R, Moskowitz L, Quencer RM. Nasopharyngeal angiofibromas: staging and management. Annals Oto Rhino Laryngology. 1984; 93:322-329.

8. Andrews JC, Fisch U, Valavanis A, Aeppli U, Makek MS. The surgical management of extensive nasopharyngeal angiofibromas with the infratemporal fossa approach. Laryngoscope. 1989; 99:429-437.

9. Radkowski D, McGill T, Healy GB, et al. Angiofibroma. Changes in staging and treatment. Arch Otolaryngology Head Neck Surg. 1996; 122:122-129.

10. WM Mendenhall, JW Werning, RW Hinerman et al. Juvenile Nasopharyngeal Angiofibroma, J HK Coll Radiol 2003;6:15-19.

11. Mojtaba Mohammadi Ardehali, Jahangir Ghorbani.Juvenile Nasophryngeal Angiofibroma, New Aspects in Management. Iranian Journal of Otorhinolaryngology No.3, Vol.23, Serial No.64, Summer-2011

12. Tobias Alecio Mattei, Gustavo Fabiano Nogueira, Ricardo Ramina. Juvenile nasopharyngeal angiofibroma with intracranial extension, Otolaryngology -- Head and Neck Surgery 2011 145: 498 originally published online 13 May 2011.

13. Shaheen, H. B.: Nasopharyngeal Fibroma. J. Laryng. and Otol., 45: 259-264, 1930.

14. Teddy Charles Adias, Zacheus Jeremiah.Autologous blood transfusion - a review. SAJS Vol 44, No. 3, August 2006:114-118. 


\section{ORIGINAL ARTICLE}

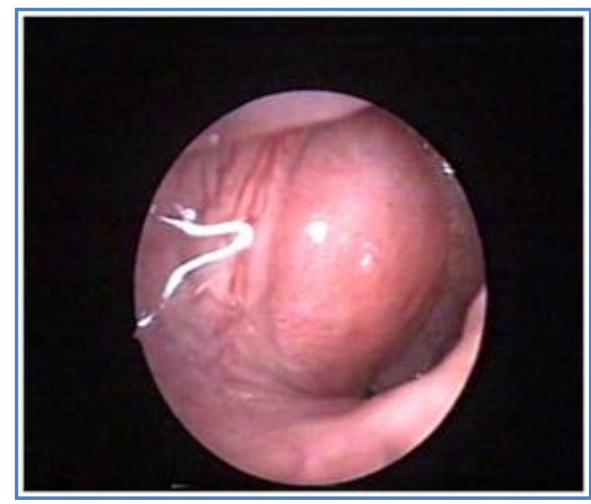

Fig. 1: Diagnostic nasal endoscopy showing

Smooth surfaced mass in the nasal cavity with prominent vessels.

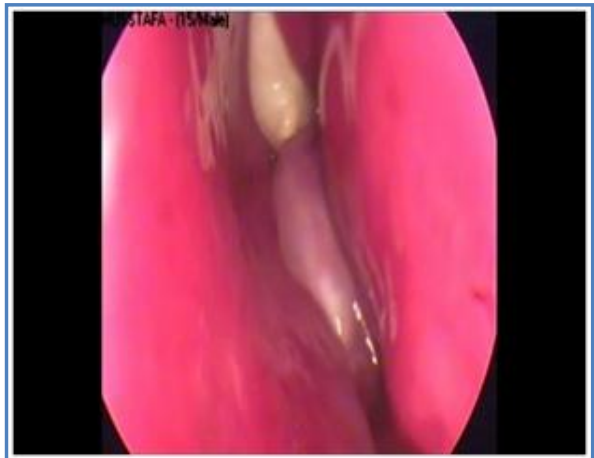

Fig. 2: Endoscopic view of a unilateral smooth surfaced mass in the left nasal cavity

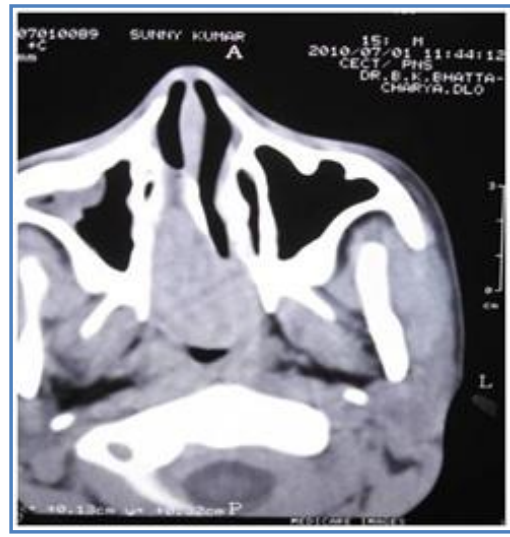

Fig. 4: CT PNS axial cut; the tumor is limited to the pterygomaxillary fissure.

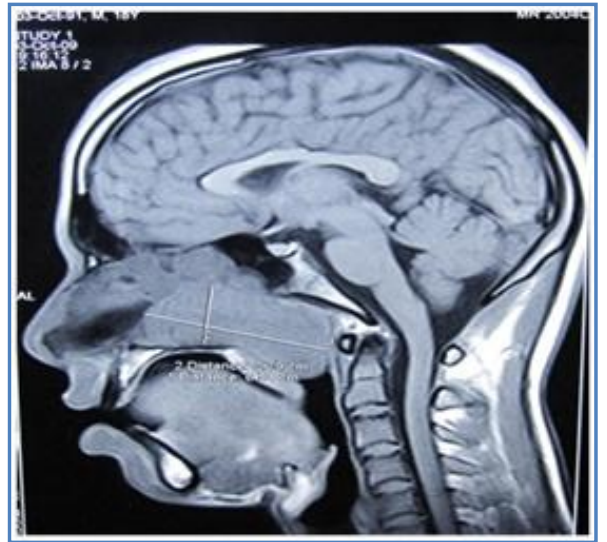

Fig. 3: MRI T1 weighted image, sagittal section showing tumor in the nasal cavity extending to nasopharynx

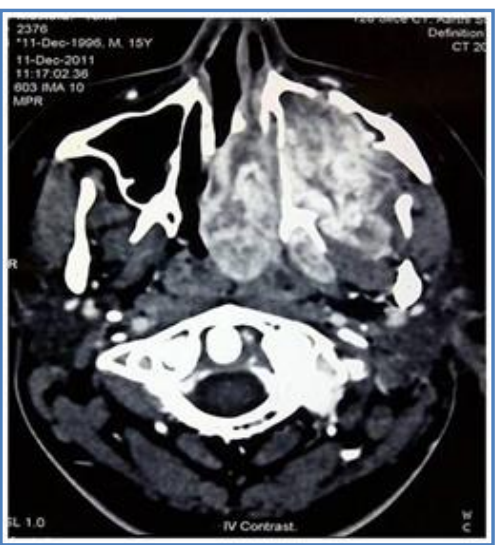

Fig. 5: CT PNS, axial section showing contrast enhanced mass extending to maxillary sinus an infratemporal fossa. 


\section{ORIGINAL ARTICLE}

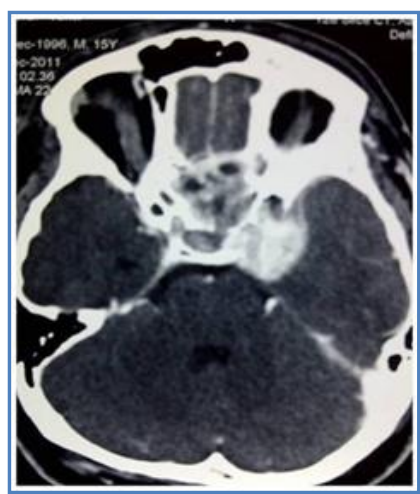

Fig. 6: CT PNS axial section showing contrast enhancingtumour occupying the middle cranial fossa.

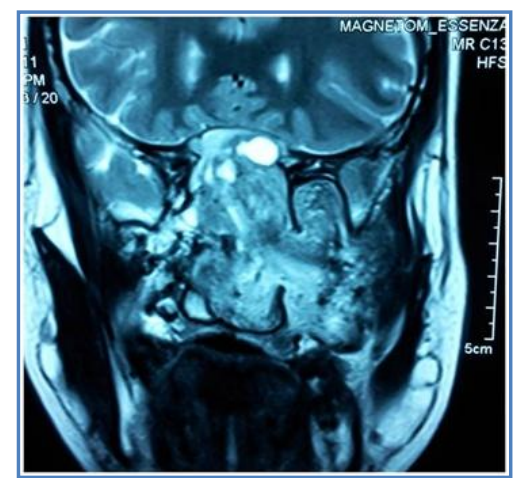

Fig. 7: MRI T2 weighed image, coronal section showing the tumor extendinginto the maxillary sinus, infratemporal fossa, sphenoid sinus and intracranial

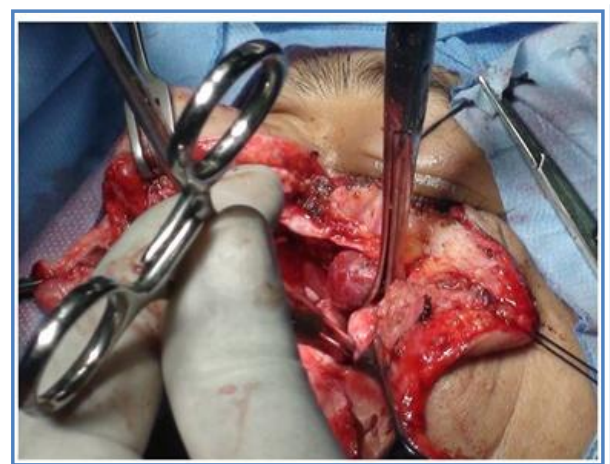

Fig. 8a: Intraoperative photo of the tumor being removed by Shaheens procedure.



Fig 8 b: Showing the entire tumor excised in toto, the various extension of the tumor is marked.

\section{AUTHORS:}

1. Prasanna Kumar S.

2. A. Ravikumar

3. Somu L.

4. Srinivasa Reddy

\section{PARTICULARS OF CONTRIBUTORS:}

1. Associate Professor, Department of ENT, Sri Ramachandra Medical College and RI, Porur, Chennai.

2. Professor, Department of ENT, Sri Ramachandra Medical College and RI, Porur, Chennai.

3. Professor, Department of ENT, Sri Ramachandra Medical College and RI, Porur, Chennai.
4. Senior Resident, Department of ENT, Sri Ramachandra Medical College and RI, Porur, Chennai.

\section{NAME ADDRESS EMAIL ID OF THE CORRESPONDING AUTHOR:}

Dr.Prasanna Kumar S.,

Associate Professor, Dept of ENT,

Head and Neck Surgery,

Sri Ramacnandra medical college and RI, Porur , Chennai

Email-sprasannakumar10@gmail.com

Date of Submission: 23/12/2013.

Date of Peer Review: 24/12/2013.

Date of Acceptance: 04/01/2014.

Date of Publishing: 14/01/2014 